2 New insights in the endocrine disruption effects of three primary metabolites of

\section{organophosphate flame retardants}

$$
\text { Quan Zhang }^{1 *} \text {, Chang Yu }{ }^{1} \text {, Lili Fu }{ }^{1} \text {, Sijia Gu}{ }^{1} \text {, Cui Wang }{ }^{2 *}
$$

1. Key Laboratory of Microbial Technology for Industrial Pollution Control of

Zhejiang Province, College of Environment, Zhejiang University of Technology,

Hangzhou, Zhejiang, 310032, China.

2. College of Life Science, Zhejiang Chinese Medical University, Hangzhou 310053,

China.

0

.

2

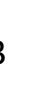

14

(1)

(1)

20

*To whom correspondence should be addressed.

Phone: +86-571- 88871579 .

E-mail address: quanzhang@zjut.edu.cn, wangcui198506@163.com 


\begin{tabular}{|c|c|}
\hline The contents of supporting information & Page \\
\hline S.1. Cells culture and plasmids & S3 \\
\hline S.2. Dual-luciferase reporter gene assay for ER and MR & S3 \\
\hline Table S1. Information on three metabolites of organophosphate flame retardants & S4 \\
\hline Table S2. The primer sequences used for qRT-PCR of zebrafish & S5 \\
\hline Table S3. The prime sequence of detected genes of H295R cells. & S6 \\
\hline $\begin{array}{l}\text { Table S4. The fold changes of } 10 \text { steroidogenic genes in H295R cells exposed to various } \\
\text { concentrations of BCIPP, BDCIPP and DPHP for } 24 \mathrm{~h}\left({ }^{*} p<0.05 \text { compared with the value }\right. \\
\text { of DMSO control). }\end{array}$ & S7 \\
\hline Table S5 Docking scores of BCIPP, BDCIPP, DPHP for ER and MR. & S8 \\
\hline $\begin{array}{l}\text { Table S6. The fold changes of } 5 \text { genes of hypothalamic-pituitary-gonadal (HPG) axis in } \\
\text { zebrafish exposed to various concentrations of BCIPP, BDCIPP and DPHP for } 96 \mathrm{~h}\left({ }^{*} p<\right. \\
0.05 \text { compared with the value of DMSO control). }\end{array}$ & S9 \\
\hline $\begin{array}{l}\text { Table S7. The fold changes of } 8 \text { genes of hypothalamic-pituitary-adrenal (HPA) axis in } \\
\text { zebrafish exposed to various concentrations of BCIPP, BDCIPP and DPHP for } 96 \mathrm{~h}\left({ }^{*} p<\right. \\
0.05 \text { compared with the value of DMSO control). }\end{array}$ & S10 \\
\hline $\begin{array}{l}\text { Figures S1. Dose response curves of } E R \text { and } M R \text {. Firefly luciferase activity was } \\
\text { normalized based on the Renilla luciferase activity. Results were presented as the fold of } \\
\text { control. Each value represents the mean } \pm \mathrm{SD} \text { of at least three independent experiments } \\
\left(\mathrm{n}=3 \text { samples, }{ }^{*} p<0.05\right) \text {. The dose response curves were fitted by logistic model. }\end{array}$ & S11 \\
\hline $\begin{array}{l}\text { Figures S2. The cytotoxicity of three metabolites of organophosphate flame retardants } \\
\text { examined in MTS assay. A: the cytotoxicity of CHO cells, B: the cytotoxicity of H295R } \\
\text { cells. }\end{array}$ & S12 \\
\hline $\begin{array}{l}\text { Figures S3. The antagonist activities of the three OPFRs metabolites to ER } \alpha \text { and agonist } \\
\text { activities to MR by dual-luciferase reporter gene assay. Firefly luciferase activity was } \\
\text { normalized based on the Renilla luciferase activity. The positive control was defined as } \\
100 \% \text {. Results were presented as the percentage quotient of the response of metabolites to } \\
\text { positive control. Each value represents the mean } \pm \text { SD of at least three independent } \\
\text { experiments }\left(\mathrm{n}=3 \text { samples, }{ }^{*} p<0.05\right) \text {. }\end{array}$ & S13 \\
\hline $\begin{array}{l}\text { Figures S4. Proportion of short tail (st) of zebrafish larvae exposed to three metabolites of } \\
\text { organophosphate flame retardants at } 10^{-5} \mathrm{M} \text { (96hpf). The results are expressed as mean } \pm \\
\text { SD of three replicates. }\end{array}$ & S14 \\
\hline
\end{tabular}


Materials and Methods

S.1 Cells culture and plasmids

The rat estrogen receptor ( $r \mathrm{ER}$ ) expression plasmid $r E R \alpha / p C I$ and the response element $p E R E-A U G-L u c+$ were kindly provided by Dr. M. Takeyoshi (Chemicals Assessment Center, Chemicals Evaluation and Research Institute, Oita, Japan). The human mineralocorticoid receptor ( $h \mathrm{MR}$ ) expression plasmid EGFP-Cl-hMR was kindly provided by Dr. Claudia Großmann (Julius Bernstein Institute for Physiology, Martin Luther University, Halle, Germany) and Dr. Jing Liu (Zhejiang University, Hangzhou, China). The plasmid $p R L-T K$ (Promega, Madison, WI) was used as an internal control in the dual-luciferase reporter gene assay.

S.2. Dual-luciferase reporter gene assay for ER and MR

The plasmids proportions in each well to detect ER, MR activity are as follows: ER activity: $r E R \alpha p C I 25 \mathrm{ng}+p E R E-A U G-L u c+145 \mathrm{ng}+p R L-T K 10 \mathrm{ng}$; MR activity: EGFP-C1-hMR $20 \mathrm{ng}+$ pMMTV-luc $160 \mathrm{ng}+p R L-T K 10 \mathrm{ng}$; 
Table S1 Information on three metabolites of organophosphate flame retardants

chemicals and abbreviation
$\begin{gathered}\text { Bis (1-chloro-2-propyl) phosphate } \\ \text { (BCIPP) }\end{gathered}$
$\begin{gathered}\text { Bis (1,3-dichloro-2-propyl) phosphate } \\ \text { (BDCIPP) } \\ \text { Diphenyl phosphate } \\ \text { (DPHP) }\end{gathered}$


Table S2. The primer sequences used for qRT-PCR of zebrafish

\begin{tabular}{|c|c|c|}
\hline Genes & Primers sequence (5'-3') & Accession number \\
\hline \multirow{2}{*}{$\beta$-actin } & F: CTGCTATGTGGCTCTTGACT & \multirow{2}{*}{ EF597101.1 } \\
\hline & R: CAGGTCCTTACGGATGTCG & \\
\hline \multirow{2}{*}{$E R \alpha$} & F: TGGGCTAAGAAAGTACCA & \multirow{2}{*}{ HМ045496.1 } \\
\hline & R: CTTGAGTTTGAGACTGCGGAAT & \\
\hline \multirow{2}{*}{$V T G$} & F: AGGGAGTATGCAGGACC & \multirow{2}{*}{ JN004056.1 } \\
\hline & R: CTCAGTGTATGCCAAACCAAT & \\
\hline \multirow{2}{*}{ CYP17 } & F: AGGGACCCGAGTCATTAT & \multirow{2}{*}{ JN858107.1 } \\
\hline & R: GGGCAGCACAAACCATCAC & \\
\hline \multirow{2}{*}{ Cyp 19a } & F: GGCTACAAAGTGAAG & \multirow{2}{*}{ GU220394.1 } \\
\hline & R: GAACGGCTGAAAGAAACGAC & \\
\hline \multirow{2}{*}{ Cyp $19 b$} & F: CCTGGTGACCCTGTTGT & \multirow[t]{2}{*}{ GU220393.1 } \\
\hline & R: GTGGCTGTCCGTCGTTTCT & \\
\hline \multirow{2}{*}{ StAR } & F: CTGAGAATGGACCCACCTGT & \multirow{2}{*}{ NM_131663 } \\
\hline & R: GCAATAAACGTCAGCAAGCA & \\
\hline \multirow{2}{*}{$H M G R A$} & F: GAGCCATCGACTCTCTCCTG & \multirow{2}{*}{$\mathrm{BC} 155135$} \\
\hline & R: GAACACGACTGCTAGCACCA & \\
\hline \multirow{2}{*}{$M R$} & F: ATTGGGCCTAGTGCAAAATG & \multirow[t]{2}{*}{ EF567113 } \\
\hline & R: TCTCTGTTTGGCTCGGTCTT & \\
\hline \multirow{2}{*}{$P O M C$} & F: AGGTCGACTATCCGCAAGAA & \multirow{2}{*}{ AY 158003} \\
\hline & R: CAACCTCTCCCCCTTAAAGC & \\
\hline \multirow{2}{*}{$C R H$} & F: TTCGGGAAGTAACCACAAGC & \multirow[t]{2}{*}{ NM_001007379 } \\
\hline & R: CTGCACTCTATTCGCCTTCC & \\
\hline \multirow{2}{*}{$3 \beta H S D$} & F: AGAGACCCGGAGAAAAGAGC & \multirow[t]{2}{*}{ AY279108 } \\
\hline & R: GGGTGGAGTGAATCTCAGGA & \\
\hline \multirow{2}{*}{ CRHR2 } & F: AAAGATGCTGGTTTGGGAAG & \multirow{2}{*}{ XM_681362 } \\
\hline & R: CCCAGTAAAGGCAGAAGCAC & \\
\hline
\end{tabular}


Table S3. The prime sequence of detected genes of H295R cells.

\begin{tabular}{cll}
\hline Genes & Primers sequence (5'-3') & Accession number \\
\hline \multirow{2}{*}{ CYP11A1 } & F: GAGATGGCACGCAACCTGAAG & NM_001099773 \\
& R: CTTAGTGTCTCCTTGATGCTGGC & \\
CYP11B1 & F: GGTTTGCCAGGCTAAGC & NM_000497 \\
& R: CAAACTGCCCAGAGGACAG & \\
CYP11B2 & F: TCCAGGTGTGTTCAGTAGTTCC & NM_000498 \\
& R: GAAGCCATCTCTGAGGTCTGTG & \\
CYP17 & F: GGCACCAAGACTACAGTGATTGG & M14564 \\
& R: AGAGTCAGCGAAGGCGATAC & \\
CYP19 & F: GCCAGTGAGGAGCAGGAC & M74714.1 \\
& R: TTGGAAATGCTGAACCCGATAC & \\
CYP21 & F: ACCTCAGTTTCTCCTTTATTGC & NM_000500 \\
& R: AGAGCCAGGGTCCTTCAC & \\
GAPDH & F: GAAGGTGAAGGTCGGAGT & NC_000012 \\
& R: GAAGATGGTGATGGGATTTC & \\
HMGR & F: TTCAGGTTCCAATGGCAACAAC & NM_000859 \\
& R: GCCACGAGTCATCCCATCTG & \\
$3 \beta H S D$ & F: AGCATCTTCTGTTTCCTGGCA & NM_000198 \\
& R: TCTCCTTCAGTTCCTTCTCTTC & \\
F $17 \beta H S D$ & F: AAGACTTGCTTGCTGTGG & KF742611 \\
& R: TTCATGGAGAAGGTGTTGG & \\
STAR & F: ATGAGTAAAGTGGTCCCAGATG & NM_000349 \\
& R: ACCTTGATCTCCTTGACATTGG & \\
\hline & &
\end{tabular}


Table S4 The fold changes of 10 steroidogenic genes in H295R cells exposed to various concentrations of BCIPP, BDCIPP and DPHP for $24 \mathrm{~h}\left({ }^{*} p<0.05\right.$ compared with the value of DMSO control).

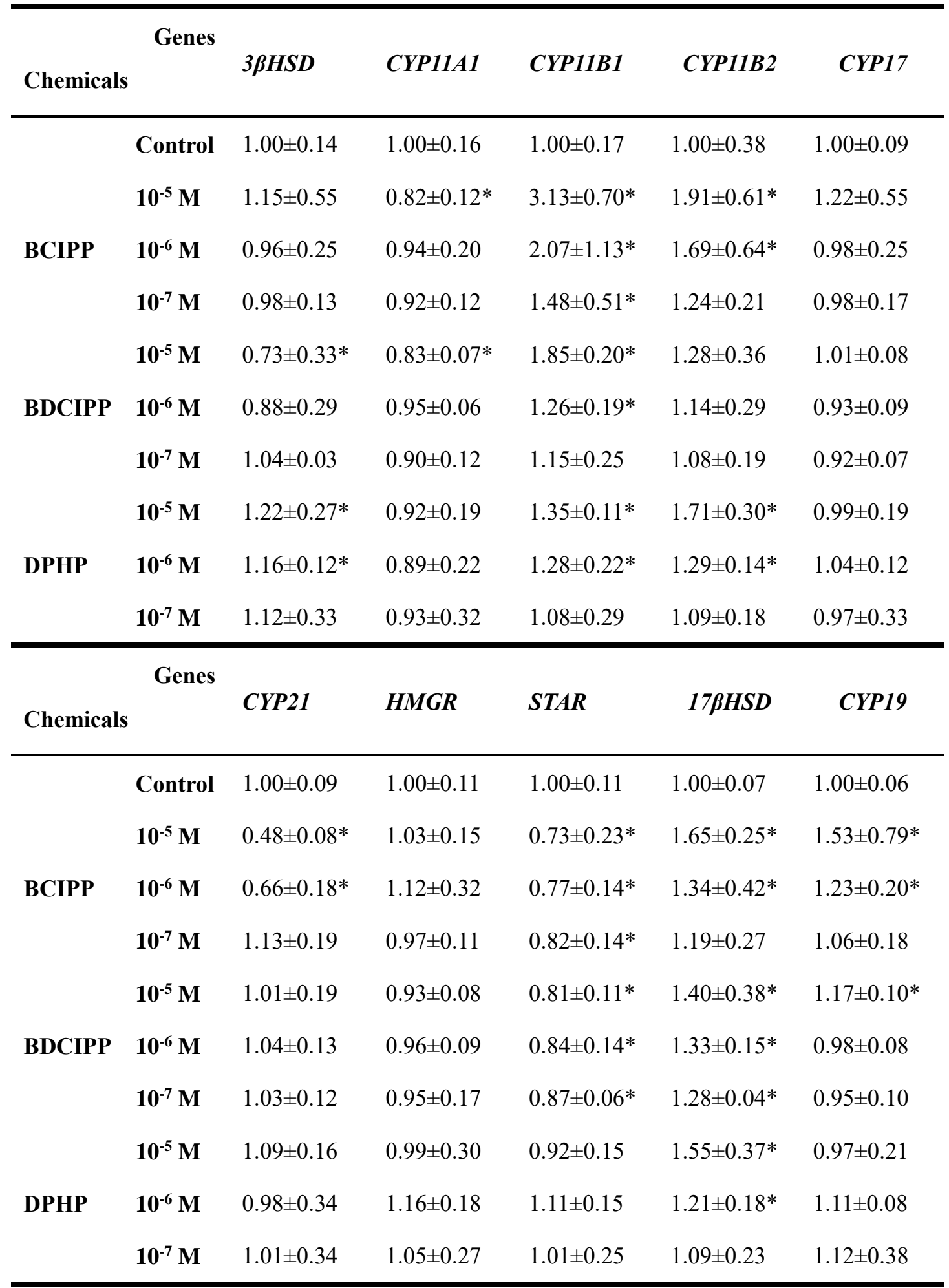


Table S5 Docking scores of BCIPP, BDCIPP, DPHP for ER and MR.

\begin{tabular}{llll}
\hline Compounds & BCIPP & BDCIPP & DPHP \\
\hline Score for ER & -8.7 & -7.3 & -6.9 \\
Score for MR & -7.6 & -8.8 & -6.7 \\
\hline
\end{tabular}


Table S6 The fold changes of 5 genes of hypothalamic-pituitary-gonadal (HPG) axis in zebrafish exposed to various concentrations of BCIPP, BDCIPP and DPHP for $96 \mathrm{~h}$ $\left({ }^{*} p<0.05\right.$ compared with the value of DMSO control).

\begin{tabular}{|c|c|c|c|c|c|c|}
\hline Chemicals & Genes & $E R \alpha$ & $V T G$ & CYP17 & Сур19a & Суp19b \\
\hline & Control & $1.00 \pm 0.06$ & $1.00 \pm 0.06$ & $1.00 \pm 0.06$ & $1.00 \pm 0.41$ & $1.00 \pm 0.05$ \\
\hline \multirow{3}{*}{ BCIPP } & $10^{-5} \mathrm{M}$ & $3.61 \pm 0.77^{*}$ & $3.66 \pm 0.44^{*}$ & $7.14 \pm 0.71 *$ & $11.14 \pm 0.98^{*}$ & $15.54 \pm 3.37 *$ \\
\hline & $10^{-6} \mathrm{M}$ & $1.29 \pm 0.07 *$ & $2.12 \pm 0.57$ & $5.80 \pm 2.03 *$ & $1.54 \pm 0.004$ & $1.70 \pm 0.09 *$ \\
\hline & $10^{-7} \mathrm{M}$ & $0.79 \pm 0.41$ & $1.73 \pm 0.48$ & $0.71 \pm 0.23$ & $0.95 \pm 0.19$ & $0.98 \pm 0.14$ \\
\hline \multirow{3}{*}{ BDCIPP } & $10^{-5} \mathrm{M}$ & $0.20 \pm 0.17^{*}$ & $10.79 \pm 5.03 *$ & $9.02 \pm 0.28 *$ & $0.97 \pm 0.16$ & $5.27 \pm 0.55^{*}$ \\
\hline & $10^{-6} \mathrm{M}$ & $0.58 \pm 0.23 *$ & $2.26 \pm 0.48^{*}$ & $2.90 \pm 1.33^{*}$ & $0.94 \pm 0.09$ & $2.67 \pm 0.31^{*}$ \\
\hline & $10^{-7} \mathrm{M}$ & $0.65 \pm 0.29$ & $1.78 \pm 0.65$ & $0.22 \pm 0.16^{*}$ & $0.82 \pm 0.40$ & $2.69 \pm 0.22^{*}$ \\
\hline \multirow{3}{*}{ DPHP } & $10^{-5} \mathrm{M}$ & $0.04 \pm 0.006^{*}$ & $2.07 \pm 0.14^{*}$ & $0.25 \pm 0.03 *$ & $0.83 \pm 0.29$ & $0.88 \pm 0.16$ \\
\hline & $10^{-6} \mathrm{M}$ & $0.83 \pm 0.77$ & $1.71 \pm 0.57$ & $0.33 \pm 0.07 *$ & $0.92 \pm 0.14$ & $1.20 \pm 0.25$ \\
\hline & $10^{-7} \mathrm{M}$ & $0.75 \pm 0.44$ & $1.78 \pm 0.30$ & $0.39 \pm 0.05 *$ & $1.44 \pm 0.29$ & $1.77 \pm 0.16$ \\
\hline
\end{tabular}


Table S7 The fold changes of 8 genes of hypothalamic-pituitary-adrenal (HPA) axis in zebrafish exposed to various concentrations of BCIPP, BDCIPP and DPHP for $96 \mathrm{~h}$ $\left({ }^{*} p<0.05\right.$ compared with the value of DMSO control).

\begin{tabular}{|c|c|c|c|c|c|}
\hline & $\begin{array}{l}\text { Genes } \\
\text { cals }\end{array}$ & $G R$ & $M R$ & STAR & $3 \beta H S D$ \\
\hline \multirow{4}{*}{$\stackrel{\square}{\sigma}$} & Control & $1.00 \pm 0.10$ & $1.00 \pm 0.02$ & $1.00 \pm 0.24$ & $1.00 \pm 0.15$ \\
\hline & $10^{-5} \mathrm{M}$ & $3.07 \pm 0.36^{*}$ & $3.67 \pm 0.89^{*}$ & $1.50 \pm 0.63$ & $3.89 \pm 0.32 *$ \\
\hline & $10^{-6} \mathrm{M}$ & $0.79 \pm 0.04$ & $1.64 \pm 0.32$ & $0.76 \pm 0.01$ & $1.51 \pm 0.35$ \\
\hline & $10^{-7} \mathrm{M}$ & $0.69 \pm 0.04$ & $0.86 \pm 0.23$ & $1.55 \pm 0.19$ & $0.98 \pm 0.04$ \\
\hline \multirow{3}{*}{ 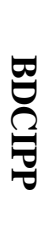 } & $10^{-5} \mathrm{M}$ & $0.56 \pm 0.02 *$ & $0.47 \pm 0.05^{*}$ & $0.72 \pm 0.06$ & $2.10 \pm 0.48$ \\
\hline & $10^{-6} \mathrm{M}$ & $0.87 \pm 0.24$ & $0.47 \pm 0.25^{*}$ & $0.73 \pm 0.30$ & $2.16 \pm 0.21$ \\
\hline & $10^{-7} \mathrm{M}$ & $0.97 \pm 0.20$ & $0.48 \pm 0.11 *$ & $0.78 \pm 0.11$ & $2.06 \pm 0.49$ \\
\hline \multirow{3}{*}{$\begin{array}{l}\underline{\theta} \\
\underline{\theta} \\
\underline{\theta}\end{array}$} & $10^{-5} \mathrm{M}$ & $3.68 \pm 0.08^{*}$ & $2.17 \pm 0.13^{*}$ & $0.22 \pm 0.02 *$ & $2.82 \pm 0.17 *$ \\
\hline & $10^{-6} \mathrm{M}$ & $0.89 \pm 0.08$ & $1.64 \pm 0.30$ & $1.93 \pm 0.74$ & $2.13 \pm 0.34$ \\
\hline & $10^{-7} \mathrm{M}$ & $1.32 \pm 0.18$ & $2.00 \pm 0.50$ & $1.65 \pm 0.52$ & $1.18 \pm 0.39$ \\
\hline & $\begin{array}{l}\text { Genes } \\
\text { als }\end{array}$ & $\mathrm{CRH}$ & CRHR2 & HMGRA & POMC \\
\hline \multirow{4}{*}{$\stackrel{\square}{\mathscr{\sigma}}$} & Control & $1.00 \pm 0.06$ & $1.00 \pm 0.30$ & $1.00 \pm 0.18$ & $1.00 \pm 0.37$ \\
\hline & $10^{-5} \mathrm{M}$ & $4.02 \pm 1.08^{*}$ & $1.45 \pm 0.05$ & $1.14 \pm 0.66$ & $0.64 \pm 0.14$ \\
\hline & $10^{-6} \mathrm{M}$ & $1.38 \pm 0.50$ & $0.81 \pm 0.15$ & $1.13 \pm 0.17$ & $0.67 \pm 0.20$ \\
\hline & $10^{-7} \mathrm{M}$ & $1.15 \pm 0.37$ & $0.84 \pm 0.08$ & $0.90 \pm 0.02$ & $1.42 \pm 0.34$ \\
\hline \multirow{3}{*}{$\begin{array}{l}\widetilde{\sigma} \\
\widetilde{\theta} \\
\underline{\partial}\end{array}$} & $10^{-5} \mathrm{M}$ & $2.57 \pm 0.36^{*}$ & $0.80 \pm 0.10$ & $0.70 \pm 0.10$ & $0.72 \pm 0.13$ \\
\hline & $10^{-6} \mathrm{M}$ & $0.96 \pm 0.03$ & $1.48 \pm 0.35$ & $1.30 \pm 0.11$ & $0.83 \pm 0.13$ \\
\hline & $10^{-7} \mathrm{M}$ & $1.15 \pm 0.20$ & $0.74 \pm 0.20$ & $0.89 \pm 0.15$ & $0.99 \pm 0.22$ \\
\hline \multirow{3}{*}{$\underset{\nabla}{\underline{\theta}}$} & $10^{-5} \mathrm{M}$ & $2.24 \pm 0.12 *$ & $0.93 \pm 0.26$ & $0.83 \pm 0.20$ & $0.84 \pm 0.23$ \\
\hline & $10^{-6} \mathrm{M}$ & $1.48 \pm 0.03 *$ & $0.82 \pm 0.09$ & $1.56 \pm 0.20$ & $0.92 \pm 0.13$ \\
\hline & $10^{-7} \mathrm{M}$ & $1.03 \pm 0.61$ & $1.55 \pm 0.21$ & $1.00 \pm 0.18$ & $1.00 \pm 0.37$ \\
\hline
\end{tabular}


ER

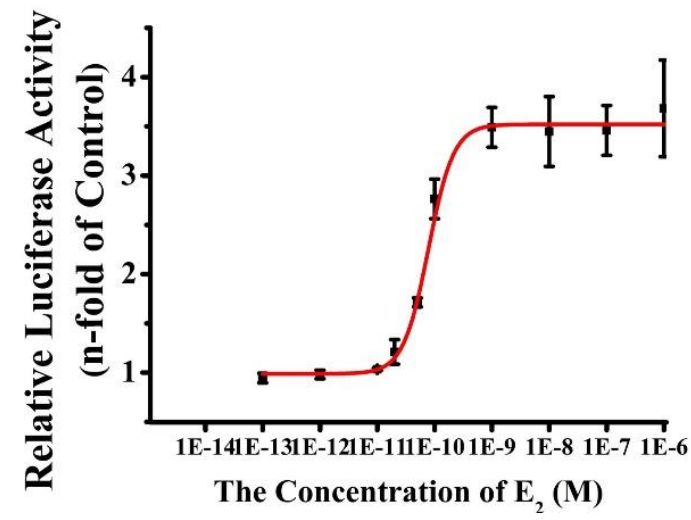

MR

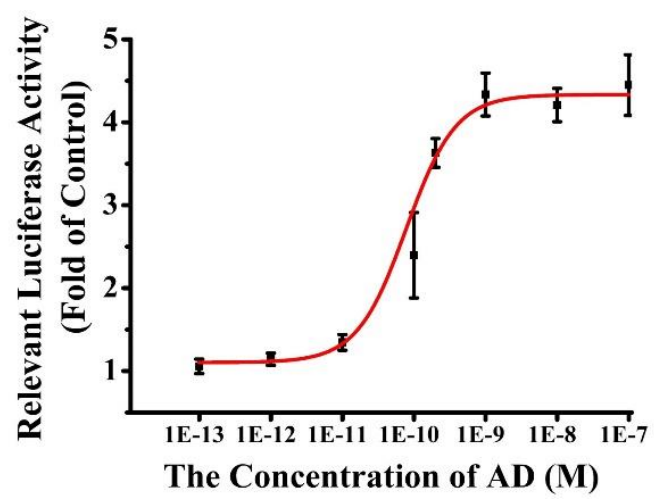

Figures S1 Dose response curves of $E_{2}$ and $A D$ obtained from the ER and MR transactivation assays. Firefly luciferase activity was normalized based on the Renilla luciferase activity. Results were presented as the fold of control. Each value represents the mean $\pm \mathrm{SD}$ of at least three independent experiments ( $\mathrm{n}=3$ samples, $\left.{ }^{*} p<0.05\right)$. The dose response curves were fitted by logistic model. 


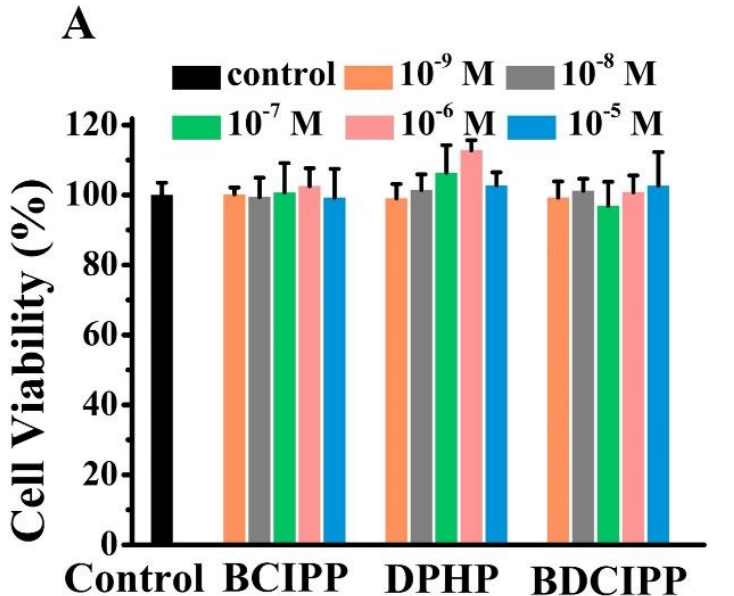

The concentration $(M)$

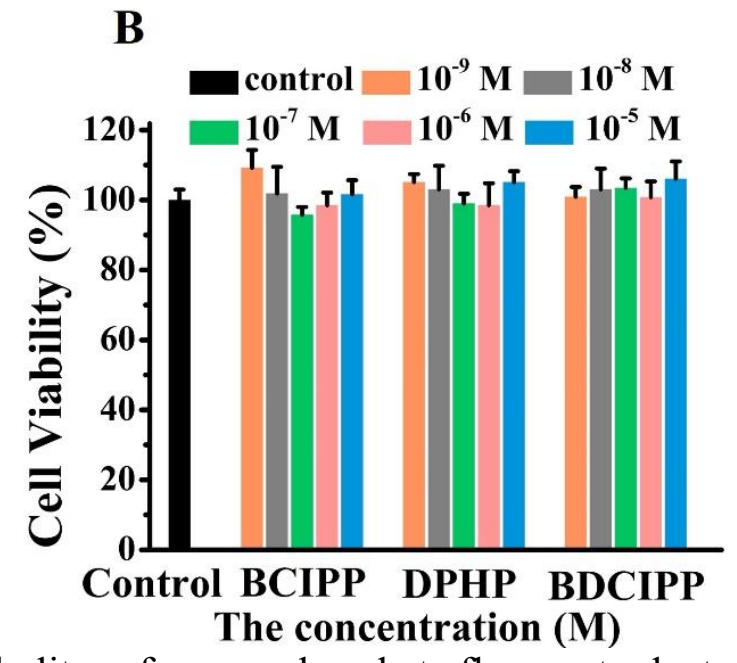

Figures S2 The cytotoxicity of three metabolites of organophosphate flame retardants

examined in MTS assay. A: the cytotoxicity of $\mathrm{CHO}$ cells, B: the cytotoxicity of H295R cells. 

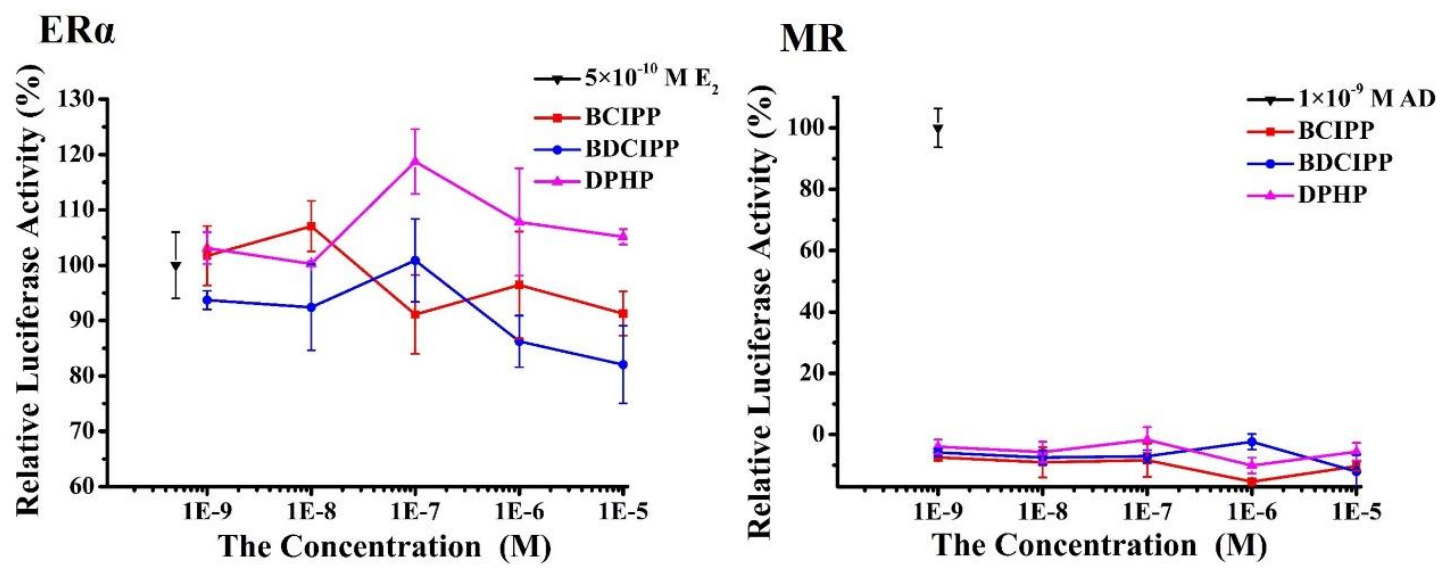

Figures S3 The antagonist activities of the three OPFRs metabolites to ER $\alpha$ and agonist activities to MR by dual-luciferase reporter gene assay. Firefly luciferase activity was normalized based on the Renilla luciferase activity. The positive control was defined as $100 \%$. Results were presented as the percentage quotient of the response of metabolites to positive control. Each value represents the mean $\pm \mathrm{SD}$ of at least three independent experiments ( $\mathrm{n}=3$ samples, $\left.{ }^{*} p<0.05\right)$. 


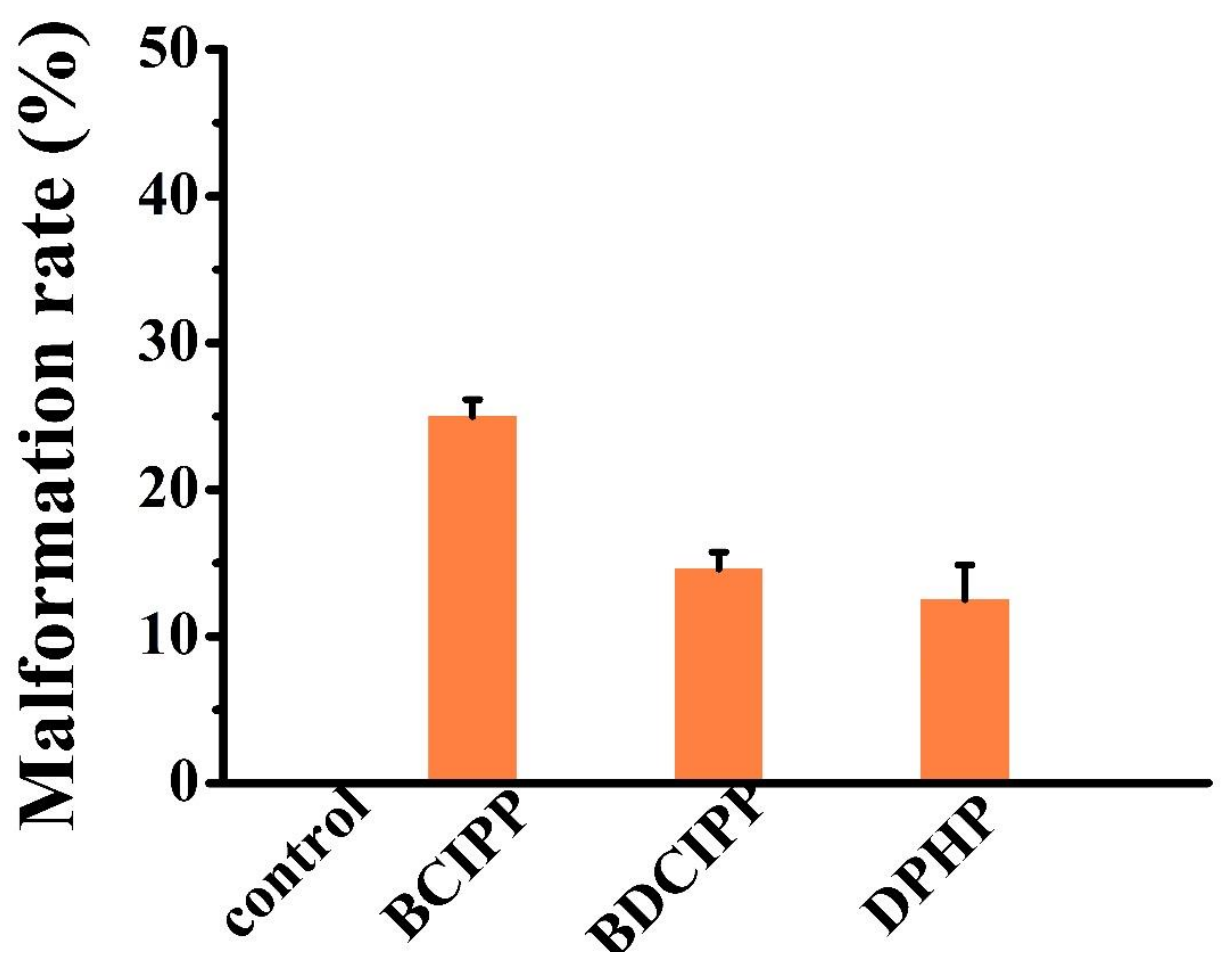

Figures S4 Proportion of short tail (st) of zebrafish larvae exposed to three metabolites of organophosphate flame retardants at $10^{-5} \mathrm{M}$ (96hpf). The results are expressed as mean $\pm \mathrm{SD}$ of three replicates. 